\title{
Soluble VEGF receptor-2 may be a predictive marker of anti-angiogenic therapy with clinically available safe agents
}

\author{
HITOSHI YOSHIJI, RYUICHI NOGUCHI, YASUHIDE IKENAKA, KOSUKE KAJI, YUSAKU SHIRAI, \\ YOSUKE AIHARA, JUNICHI YAMAO, MASAHISA TOYOHARA, AKIRA MITORO, MASAYOSHI SAWAI, \\ MOTOYUKI YOSHIDA, CHIE MORIOKA, MASAO FUJIMOTO, MASAHITO UEMURA, \\ HIDETO KAWARATANI, TATSUHIRO TSUJIMOTO and HIROSHI FUKUI
}

Third Department of Internal Medicine, Nara Medical University, Nara 634-8522, Japan

Received June 28, 2010; Accepted September 14, 2010

DOI: 10.3892/ol.2010.196

\begin{abstract}
The identification of biomarkers of anti-angiogenic therapy that predict clinical benefit is of vital importance. We previously reported that a combination treatment with clinically available safe agents, specifically angiotensin-converting enzyme inhibitor (ACE-I) and vitamin $\mathrm{K}(\mathrm{VK})$, inhibited the cumulative recurrence of hepatocellular carcinoma (HCC) via suppression of the vascular endothelial growth factor (VEGF). The present study aimed to identify non-invasive biological markers that predict the clinically beneficial effect of this combination regimen. A combination of ACE-I (perindopril; 4 $\mathrm{mg}$ /day) and VK (menatetrenone; $45 \mathrm{mg}$ /day) was administered for 54 months following curative therapy for HCC. The cumulative recurrence and several indices, which are reportedly considered as biological markers of anti-angiogenic therapies, were analyzed. The combined treatment of ACE-I and VK markedly inhibited the cumulative recurrence of HCC during the 54-month follow-up. The serum VEGF and soluble VEGF receptor (sVEGFR)-2 were significantly suppressed with this combination regimen, whereas sVEGFR-1 was not. In HCC patients without recurrence, a significant suppression of VEGF and sVEGFR-2 was achieved within 6 and 3 months after treatment, respectively. In conclusion, the combination treatment of
\end{abstract}

Correspondence to: Dr Hitoshi Yoshiji, Third Department of Internal Medicine, Nara Medical University, 840 Shijo-cho, Kashihara, Nara 634-8522, Japan

E-mail: yoshijih@naramed-u.ac.jp

Abbreviations: AT-II, angiotensin-II; ACE, angiotensin-converting enzyme; AT1-R, angiotensin type-1 receptor; ACE-I, ACE inhibitor; $\mathrm{ARB}$, angiotensin type-1 receptor blocker; EC, endothelial cell; HCC, hepatocellular carcinoma; IFN, interferon; RFA, percutaneous radiofrequency ablation; sVEGFR-2, soluble form of VEGF receptor-2; VK, vitamin K2; VEGF, vascular endothelial growth factor

Key words: vascular endothelial growth factor, soluble vascular endothelial growth factor receptor-2, angiogenesis, hepatocellular carcinoma
ACE-I and VK is a potentially novel anti-angiogenic strategy for secondary chemoprevention against HCC since the two agents are widely used in clinical practice without serious side effects. Furthermore, sVEGFR-2 may become a useful clinical predictive marker of this combination treatment.

\section{Introduction}

Hepatocellular carcinoma (HCC) is a malignancy of worldwide significance, and its prevalence is on the increase in Japan, Western Europe and the US (1). One of the reasons for the poor prognosis of $\mathrm{HCC}$ is its high rate of recurrence. Mounting evidence indicates that the high rate of recurrence, even after curative therapy, is due to intrahepatic metastasis or the multi-centric development of each respective neoplasm clone. Since the high-risk group of either primary or secondary HCC development appears to be more discernable than in other types of tumors, it is likely that chemopreventive agents are beneficial in improving the prognosis of HCC.

Any solid tumor that has not acquired a new blood supply cannot grow to more than a few millimeters in size, including HCC (2). Numerous studies showed that neovascularization and angiogenic factors, such as the vascular endothelial cell growth factor (VEGF), are significantly up-regulated in human HCC samples $(3,4)$. It has been reported that angiogenesis is also induced at the early stages of tumor formation and carcinogenesis (5-8). In a previous study, angiogenesis was found to increase stepwise during the murine hepatocarcinogenesis process and suppression of the VEGF-signaling pathway markedly attenuated hepatocarcinogenesis (9). It has been reported that alterations in the hepatic microcirculation in the human liver develop at an early stage of liver carcinogenesis, in association with liver cell change or within the dysplastic nodules, prior to the emergence of morphologically identifiable HCC (10).

A number of anti-angiogenic agents have already been employed in clinical practice, including sorafenib (11). Sorafenib is an oral multi-kinase inhibitor that acts against several tyrosine kinases (VEGFR-1, PDGFR and c-kit receptors) and serine/threonine kinases (b-Raf and p38) (12). A recent study showed that sorafenib prolonged the median overall survival and delayed the median time to progression in 
patients with advanced HCC; thus, this drug is to become the standard therapy for advanced stages of HCC (13). However, various serious concerns exist in employing this agent for chemoprevention against HCC. Since long-term administration is required and the drug metabolism is usually hypoactive in patients with cirrhosis, an agent with proven safety is preferable for chemoprevention against HCC. Almost all patients show adverse effects upon using sorafenib, and several of the symptoms are relatively severe $(14,15)$. Moreover, considering the long-term administration, cost effectiveness is an issue due to the high cost involved (16). An alternative approach may be to find a clinically available compound that also exhibits anti-angiogenic activity, for which the safety of long-term administration has been proven.

In a previous study, it was reported that the angiotensinconverting enzyme inhibitor (ACE-I) and vitamin K2 (VK) exerted strong anti-angiogenic activities. Additionally, the two agents are widely used in clinical practice as safe drugs for hypertension (ACE-I) and osteoporosis (VK). The combination treatment of ACE-I and VK at clinically comparable low doses was found to show a marked suppressive effect against the development of $\mathrm{HCC}$ in rats via angiogenesis suppression (17). The combined treatment of ACE-I and VK also ameliorated hepatic dysplastic nodules in a patient with liver cirrhosis (18). Furthermore, this combination regimen significantly inhibited the cumulative recurrence of HCC after curative therapy, at least partly, through suppression of VEGFmediated neovascularization (19).

Markers that predict clinical benefit are crucial, particularly those inhibiting VEGF, since sorafenib occasionally exerts severe diverse effects, as described above. A study recently showed that hepatocyte growth factor (HGF) may be a surrogate marker for the clinical benefit of sorafenib (20). In addition to $\mathrm{HGF}$, various molecules have been considered as useful biological markers for anti-angiogenic therapy, such as circulating endothelial progenitor cells and the soluble form of VEGF receptors (20).

In the present study, a long-term follow-up (54 months) was performed to ascertain whether the combination of ACE-I and $\mathrm{VK}$ inhibits disease recurrence in patients with $\mathrm{HCC}$ after following curative radiofrequency ablation (RFA) therapy. Furthermore, the identification of non-invasive biological markers that predict the clinical benefit of this regimen were investigated.

\section{Materials and methods}

Patients. The study comprised 54 patients with HCC who were admitted to hospital between July 2004 and July 2009. HCC was diagnosed via a combination of various imaging modalities, such as hepatic angiography, enhanced computed tomography (CT) and magnetic resonance imaging (MRI). In the present study, no unusual lesions were encountered that required needle biopsy for histological confirmation; thus, no histological proof of the diagnosis was obtained. Therapeutic modalities according to the algorithm of HCC treatment by the Liver Cancer Study Group of Japan (LCSGJ) were employed. The LCSGJ scoring system is a reliable system comparable to those of the Cancer of the Liver Italian Program, the Barcelona Clinic Liver Center and the recently published evidence-based clinical practice guidelines for HCC in Japan (supported by the Japanese Ministry of Health, Labor, and Welfare) (21-23). In this algorithm, the therapeutic strategy is selected based on the degree of liver damage as determined by LCSGJ and the characteristics of the tumor itself. The indications of RFA of LCSGJ are $\leq 3$ tumors measuring $\leq 3 \mathrm{~cm}$, or a solitary tumor with a major axis of $\leq 5 \mathrm{~cm}$. Thus, when RFA is selected as a therapeutic modality, the patient is admitted to our department.

Studydesign and treatment. The patients were randomly divided (using sealed envelopes) into either the control group (Group $1, \mathrm{G} 1 ; \mathrm{n}=28$ ) or the combination-treated group (Group 2, G2; $\mathrm{n}=26$ ). No placebo was used in $\mathrm{G} 1$. The patients in $\mathrm{G} 2$ received a constant dose of oral ACE-I (perindopril; $4 \mathrm{mg} /$ day) and VK (menatetrenone; $45 \mathrm{mg} /$ day) for 54 months. The respective doses of the two compounds are standard in clinical practice. The clinical profiles, laboratory data and patient characteristics are shown in Table I. Patients who received medication that potentially influenced the VK metabolism, such as warfarin, and those who received other types of anti-hypertensive agents were excluded from this study. Patients were also required to cease alcohol intake completely. Until the end of the recurrence follow-up, no patients received any additional therapy for HCC, including interferon (IFN). After RFA, follow-ups were conducted using enhanced CT and ultrasonography (US) once a month for the first 3 months. Detections of viable HCC during this period resulted in patient exclusion. All of the patients gave informed written consent prior to participating in the study. The study protocol was approved by the Institutional Review Board of the Nara Medical University and the study was conducted in compliance with ethical and humane principles. The present study adhered to the CONSORT statement for randomized studies.

Assessment of biological markers. The serum tumor markers of $\alpha$-fetoprotein (AFP), lectin-reactive AFP (AFP-L3) and des- $\gamma$-carboxyprothrombin (PIVKA-II), were measured every 2 months using routine laboratory methods. Prior to and at 12 months after drug administration commenced, the alterations of angiogenic factors were determined using a TranSignal Angiogenesis Antibody Array (Panomics Inc., Redwood city, CA, USA) in the serum, according to the manufacturer's manual, after equalization of the protein content. The alterations of VEGF (24) and the soluble form of VEGF receptor-1 (sVEGFR-1) and -2 (sVEGFR-2) in the serum were also examined using an enzyme-linked immunosorbent assay (ELISA) kit (R\&D Systems) according to the manufacturer's instructions as previously described (9).

Statistical analysis. Patient characteristic variables were analyzed with the Mann-Whitney U test and the Fisher's exact probability test. The cumulative recurrence of HCC was plotted using the Kaplan-Meier method, and the differences in recurrence curves were tested using the log-rank test.

\section{Results}

Patient characteristics and HCC recurrence. The clinical characteristics of the enrolled patients are shown in Table I. 
Table I. Clinical characteristics of the enrolled patients.

\begin{tabular}{lccc}
\hline Characteristics & Untreated control & VK + ACE-I & P-value \\
\hline No. of patients & 28 & 26 & $0.363^{\mathrm{b}}$ \\
Age (range; years) & $61.4 \pm 10.3^{\mathrm{a}}$ & $62.5 \pm 9.6$ & $0.491^{\mathrm{c}}$ \\
Gender (M/F) & $18 / 10$ & $19 / 7$ & $0.911^{\mathrm{c}}$ \\
Etiology (HCV/HBV/other) & $24 / 3 / 1$ & $22 / 3 / 1$ & $0.602^{\mathrm{c}}$ \\
Alcohol (<40 g/>40 g/day) & $16 / 12$ & $13 / 13$ & $0.898^{\mathrm{c}}$ \\
Tumor stage (I/II/III) & $15 / 11 / 2$ & $13 / 12 / 1$ & $0.412^{\mathrm{b}}$ \\
Tumor mean size (mm) & $21.2 \pm 8.7$ & $18.9 \pm 9.1$ & $0.068^{\mathrm{b}}$ \\
No. of tumors & $1.76 \pm 1.12$ & $1.55 \pm 0.83$ & $0.150^{\mathrm{b}}$ \\
AFP (ng/ml) & $76.5 \pm 213.3$ & $83.3 \pm 262.5$ & $0.188^{\mathrm{b}}$ \\
PIVKA-II (mAU/ml) & $52.3 \pm 60.8$ & $60.5 \pm 72.6$ & $0.329^{\mathrm{b}}$ \\
ALT (IU/l) & $71.4 \pm 33.5$ & $72.3 \pm 36.6$ & $0.897^{\mathrm{c}}$ \\
Child-Pugh score (A/B) & $23 / 5$ & $21 / 5$ & \\
\hline
\end{tabular}

${ }^{a}$ Data represents mean \pm SD. ${ }^{b, c}$ Statistical analysis was performed with the Mann-Whitney U and Fisher's exact probability tests, respectively. AFP, $\alpha$-fetoprotein; PIVKA-II, des- $\gamma$-carboxyprothrombin and ALT, alanine aminotransferase.

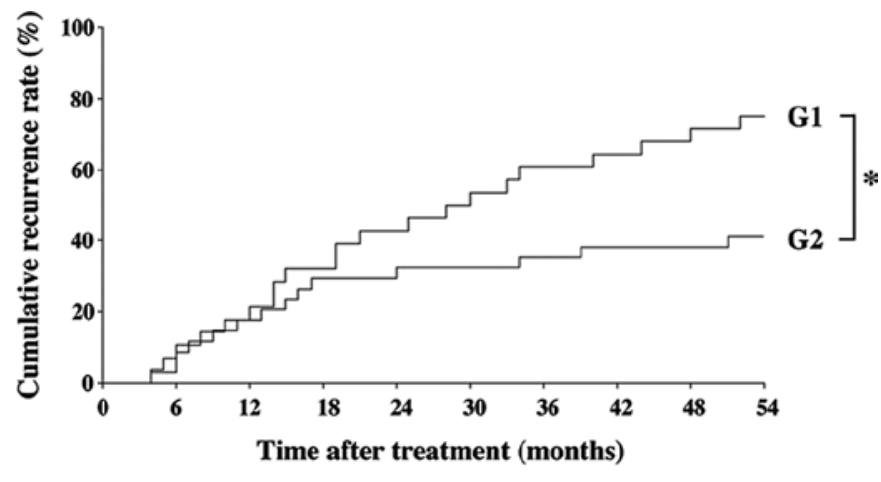

Figure 1. Cumulative recurrence of secondary HCC after curative therapy with the combination treatment of ACE-I and VK for 54 months. The combination treatment of ACE-I and VK $(\mathrm{G} 2, \mathrm{n}=26)$ significantly inhibited the cumulative recurrence of $\mathrm{HCC}$ as compared to the control group ( $\mathrm{G} 1, \mathrm{n}=28)$. *Statistically significant differences between G1 and G2 ( $<<0.01)$.

No significant differences were noted among the patients of the two groups regarding age, gender, etiology of the disease or background liver function (Child-Pugh score). In addition, the tumor baseline, such as stage, number of tumors, AFP, AFP-L3 and PIVKA-II levels, did not differ between the groups. Since no abnormal laboratory data were found that correlated to treatment with either ACE-I or VK, we were able to follow up all of the enrolled patients in each group until detection of recurrence.

The inhibitory effect of the combined treatment of ACE-I and $\mathrm{VK}$ is shown in Fig. 1. The combination treatment of ACE-I and VK (G2) significantly inhibited the cumulative recurrence of HCC as compared to the control group (G1) for 54 months after the treatment $(\mathrm{P}<0.01)$. Although the primary end-point of the study was the cumulative recurrence rates, we also examined whether or not the survival curves of the patients were altered during the follow-up period. No statistical differences were noted between the groups (data not shown).
Alteration of angiogenic factors. To elucidate which angiogenic factors were altered by treatment with ACE-I and VK, we employed the angiogenic antibody array that detects 19 different angiogenic factors in the serum of the enrolled patients following the 12-month treatment, as previously described (19). In the control group, the hot spot of VEGF was significantly increased, whereas it was markedly attenuated by the combined treatment of ACE-I and VK. One recent report showed that the HGF level may be a biomarker of sorafenib clinical efficiency. However, in our ACE-I and VK combination treatment, no significant alteration of HGF was detected (data not shown).

Plasma levels of VEGF, sVEGFR-1 and sVEGFR-2. Since VEGF expression was predominantly altered by ACE-I and VK combination treatment, we measured the expression levels of VEGF-related molecules using ELISA in all of the patients. The serum VEGF level in the control group increased after 12 months, whereas the combination treatment of ACE-I and VK markedly attenuated the VEGF level as compared to the pre-treatment level (Fig. 2A). Additionally, the expression levels of the soluble forms of sVEGFR-1 and sVEGFR-2 were elucidated. As shown in Fig. 2B, no significant differences were noted in sVEGFR-1 between the the pre-treatment and post-treatment levels in the two groups. On the other hand, sVEGFR-2 was markedly decreased by the combination treatment of ACE-I and VK, whereas the serum level of sVEGFR-2 in the control group increased (Fig. 2C). The chronological alterations of VEGF and sVEGFR-2 in the treated group were then examined until 12 months after treatment in the patients without HCC recurrence. The VEGF expression level gradually decreased, but at 6 months of treatment it was statistically significant compared to the pre-treatment basal level. The decrease in sVEGFR-2 expression occurred earlier than that of VEGF. sVEGFR-2 expression was observed to have already significantly decreased at 3 months following the ACE-I and VK combination treatment (Fig. 3). 

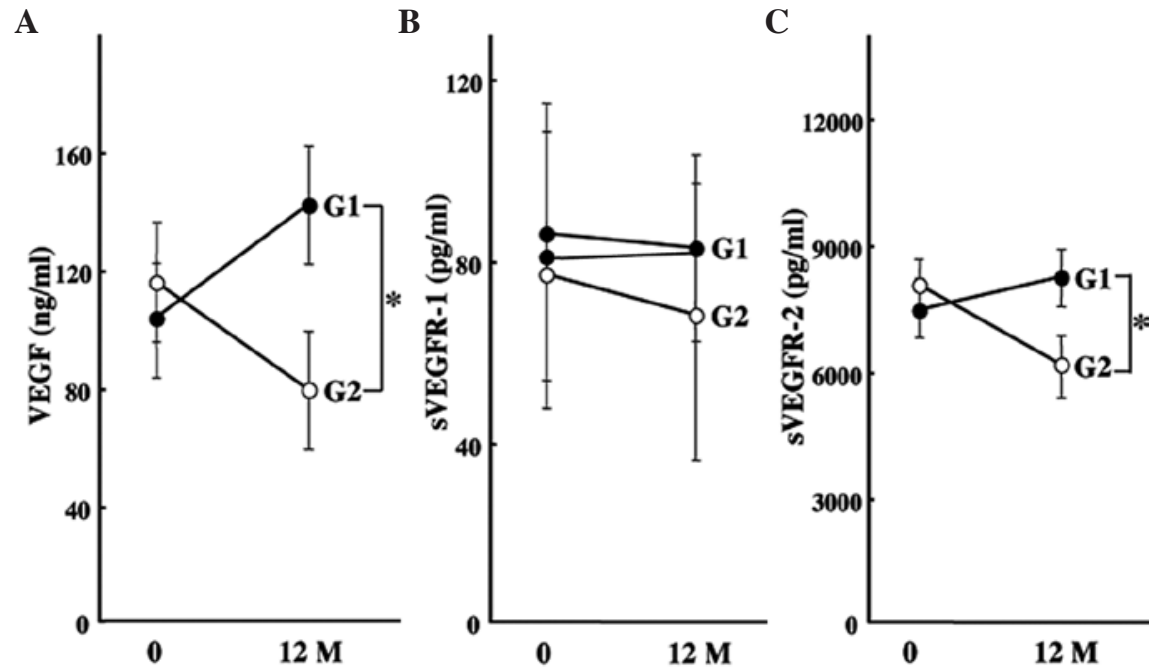

Figure 2. Effects of the combination treatment of ACE-I and VK on serum VEGF, sVEGFR-1 and sVEGFR-2 levels. (A) The serum VEGF level in the control group (G1) increased after 12 months, whereas the combination treatment of ACE-I and VK (G2) markedly attenuated the VEGF level as compared to the pre-treatment level. (B) No significant differences were noted in sVEGFR-1 between the pre-treatment and post-treatment levels in the two groups. (C) On the other hand, sVEGFR-2 was markedly decreased by the combination treatment of ACE-I and VK, whereas the serum level of sVEGFR2 in the control group increased. The data represent means $\pm \mathrm{SD}(\mathrm{n}=15)$. "Statistically significant differences between $\mathrm{G} 1$ and $\mathrm{G} 2(\mathrm{p}<0.01)$.

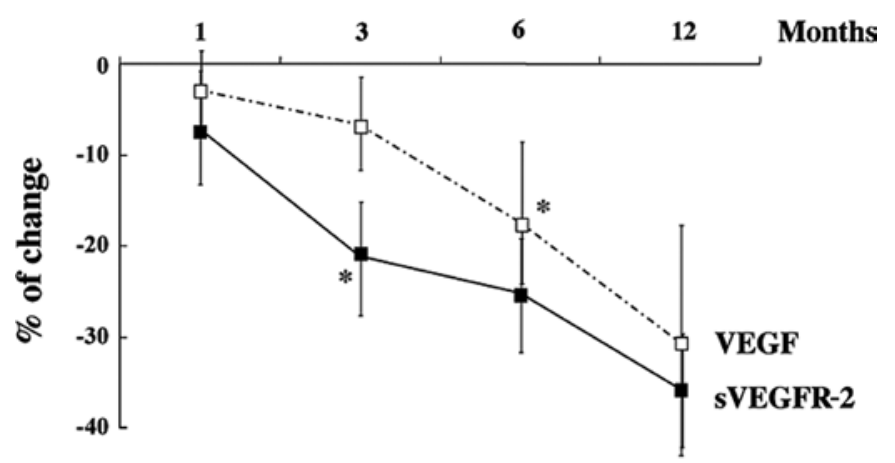

Figure 3. Chronological alterations of VEGF and sVEGFR-2 during 12 months of treatment in patients in the treated group without HCC recurrence. VEGF expression gradually decreased, and the expression level at 6 months after treatment was statistically significant as compared to the pre-treatment basal level. The decrease in sVEGFR-2 expression occurred earlier than that of VEGF. sVEGFR-2 expression was noted to have already significantly decreased at 3 months after ACE-I and VK combination treatment. The data represent the means \pm SD $(n=25)$. "Statistically significant differences as compared to the pre-treatment basal level $(\mathrm{p}<0.01)$.

\section{Discussion}

The identification of surrogate markers that predict the clinical outcome of anti-angiogenic therapies is crucial, since a number of currently available anti-angiogenic agents exert severe diverse effects. The present study showed that a combination treatment with ACE-I and VK significantly inhibited the cumulative $\mathrm{HCC}$ recurrence for 54 months after curative therapy along with the suppression of VEGF and sVEGFR-2. Since the two agents are widely used in clinical practice without serious side effects, this combined treatment is a potential new strategy for secondary chemoprevention against HCC.

One of the first biomarkers for evaluating anti-angiogenic therapy was the pre-treatment level of VEGF in tissue and circulation, but it failed to predict the outcome of anti-VEGF therapy in several types of cancer. Only the E4559 trial on non- small cell lung cancer revealed that the pre-treatment plasma VEGF level was of prognostic significance (25). In addition to VEGF, sVEGFR-1 and sVEGFR-2 have also been studied as potential biomarkers. These two full-length receptors have an extracellular domain containing seven immunoglobulinlike loops, a transmembrane domain and an intracellular split catalytic tyrosine kinase domain. sVEGFR-1 is the product of alternative mRNA splicing and comprises only six of seven immunoglobulin-like domains. sVEGFR-1 is reportedly a potential surrogate marker for disease progression in several types of cancer, such as breast cancer and renal cell carcinoma. In HCC, sVEGFR-1 was found to be correlated with poor prognosis, whereas it was not an independent prognostic factor for HCC (26). In the present study, no significant correlation was found between the alteration of sVEGFR-1 and HCC recurrence.

The natural form, sVEGFR-2, was detected more recently than sVEGFR-1. Similar to sVEGFR-1, sVEGFR-2 is unable to carry out tyrosine transphosphorylation and activate the downstream signal transduction of angiogenesis, due to the lack of the tyrosine kinase domain (20). The activation of VEGFR-2 reportedly plays a significant role in tumor angiogenesis. We previously reported that VEGF and VEGFR-2 interaction plays a pivotal role in HCC growth and hepatocarcinogenesis $(9,27)$. The neutralizing VEGFR-2 monoclonal antibody significantly attenuated hepatocarcinogenesis and HCC growth along with VEGF-mediated neovascularization. Clinical studies have evaluated the plasma levels of sVEGFR-2 as a potential surrogate marker of disease progression in various malignancies based on the hypothesis that the circulating sVEGFR-2 level may provide insight into VEGFR-2 activation (28). In the present study, it was noted that both VEGF and sVEGR-2 were significantly decreased by the combination treatment of ACE-I and VK. These results indicate that the suppressive effect of this combination regimen on $\mathrm{HCC}$ recurrence is, at least partly, mediated by the suppression of VEGF and VEGFR-2mediated neovascularization. However, no specific histological 
evidence of alteration of neovascularization in the liver was found. Although the serum VEGF level reportedly reflects the intrahepatic VEGF expression level in patients with chronic liver diseases (29), chronological immunohistochemical studies are required to confirm whether alterations of VEGF and sVEGFR-2 reflect intrahepatic neovascularization.

We observed that the expression of VEGF and sVEGFR-2 was chronologically decreased by the combined treatment of ACE-I and VK in patients without HCC recurrence. The decrease in sVEGFR-2 expression occurred earlier than that of VEGF. sVEGFR-2 expression was noted to have already significantly decreased at 3 months following ACE-I and VK combination treatment. In renal cell carcinoma, sVEGFR-2 levels were found to be significantly decreased by treatment with sunitinib, which is also a multi-kinase inhibitor, including VEGFR during treatment cycle 1 (30). Moreover, the levels tended to return to near baseline 2 weeks after treatment, suggesting that sVEGFR-2 is potentially a sensitive marker of anti-angiogenic therapy. These results show that sVEGFR-2 may be utilized as a useful clinical predictive marker of the combination treatment of ACE-I and VK.

Although the primary end-point of the study was the cumulative recurrence rates, we also examined whether the survival curves of the patients were altered or not during the follow-up period. No statistical differences were found between the groups (data not shown). Due to a short follow-up period, no statistical differences were detected between the two groups in this study. Further long-term and large-scale studies are required to verify whether or not the suppressive effects of ACE-I and VK on cumulative recurrence improve prognosis.

In conclusion, we demonstrated that the combination treatment of ACE-I and VK significantly attenuated the cumulative recurrence of HCC for 54 months following curative therapy along with the suppression of VEGF and sVEGFR-2 serum levels. In patients without recurrence, a significant suppression of VEGF and sVEGFR-2 was achieved within 6 and 3 months following treatment, respectively. These results suggest that the combination treatment of ACE-I and VK is a potential new anti-angiogenic strategy for secondary chemoprevention against $\mathrm{HCC}$, since the two agents are widely used in clinical practice without serious side effects. Furthermore, sVEGFR-2 may be a useful clinical predictive marker of this combination treatment.

\section{References}

1. Schafer DF and Sorrell MF: Hepatocellular carcinoma. Lancet 353: 1253-1257, 1999.

2. Kerbel RS: Tumor angiogenesis: past, present and the near future. Carcinogenesis 21: 505-515, 2000.

3. Guo RP, Zhong C, Shi M, et al: Clinical value of apoptosis and angiogenesis factors in estimating the prognosis of hepatocellular carcinoma. J Cancer Res Clin Oncol 132: 547-555, 2006.

4. Iavarone M, Lampertico P, Iannuzzi F, et al: Increased expression of vascular endothelial grow th factor in small hepatocellular carcinoma. J Viral Hepat 14: 133-139, 2007.

5. Li CY, Shan S, Huang Q, et al: Initial stages of tumor cellinduced angiogenesis: evaluation via skin window chambers in rodent models. J Natl Cancer Inst 92: 143-147, 2000.

6. Bergers $\mathrm{G}$ and Benjamin LE: Tumorigenesis and the angiogenic switch. Nat Rev Cancer 3: 401-410, 2003.

7. Bergers G, Javaherian K, Lo KM, Folkman J and Hanahan D: Effects of angiogenesis inhibitors on multistage carcinogenesis in mice. Science 284: 808-812, 1999.
8. Brandvold KA, Neiman P and Ruddell A: Angiogenesis is an early event in the generation of myc-induced lymphomas. Oncogene 19: 2780-2785, 2000.

9. Yoshiji H, Kuriyama S, Yoshii J, et al: Halting the interaction between vascular endothelial growth factor and its receptors attenuates liver carcinogenesis in mice. Hepatology 39: 15171524,2004

10. Frachon S, Gouysse G, Dumorti J, et al: Endothelial cell marker expression in dysplastic lesions of the liver: an immunohistochemical study. J Hepatol 34: 850-857, 2001.

11. Kerbel RS: Tumor angiogenesis. N Engl J Med 358: 2039-2049, 2008.

12. Wilhelm SM, Carter C, Tang L, et al: BAY 43-9006 exhibits broad spectrum oral antitumor activity and targets the RAF/ MEK/ERK pathway and receptor tyrosine kinases involved in tumor progression and angiogenesis. Cancer Res 64: 7099-7109, 2004.

13. Llovet JM, Ricci S, Mazzaferro V, et al: Sorafenib in advanced hepatocellular carcinoma. N Engl J Med 359: 378-390, 2008.

14. Eskens FA and Verweij J: The clinical toxicity profile of vascular endothelial growth factor (VEGF) and vascular endothelial growth factor receptor (VEGFR) targeting angiogenesis inhibitors: a review. Eur J Cancer 42: 3127-3139, 2006.

15. Verheul HM and Pinedo HM: Possible molecular mechanisms involved in the toxicity of angiogenesis inhibition. Nat Rev Cancer 7: 475-485, 2007.

16. Berenson A: A cancer drug shows promise, at a price that many can't pay. The New York Times: A1, C2, 2006.

17. Yoshiji H, Kuriyama S, Noguchi R, et al: Combination of vitamin $\mathrm{K}(2)$ and the angiotensin-converting enzyme inhibitor, perindopril, attenuates the liver enzyme-altered preneoplastic lesions in rats via angiogenesis suppression. J Hepatol 42: 687-693, 2005.

18. Yoshiji H, Noguchi R, Yamazaki M, et al: Combined treatment of vitamin $\mathrm{K} 2$ and angiotensin-converting enzyme inhibitor ameliorates hepatic dysplastic nodule in a patient with liver cirrhosis. World J Gastroenterol 13: 3259-3261, 2007.

19. Yoshiji H, Noguchi R, Toyohara M, et al: Combination of vitamin $\mathrm{K} 2$ and angiotensin-converting enzyme inhibitor ameliorates cumulative recurrence of hepatocellular carcinoma. J Hepatol 51: 315-321, 2009.

20. Murukesh N, Dive C and Jayson GC: Biomarkers of angiogenesis and their role in the development of VEGF inhibitors. Br J Cancer 102: 8-18, 2010.

21. Kokudo N and Makuuchi M: Evidence-based clinical practice guidelines for hepatocellular carcinoma in Japan: the J-HCC guidelines. J Gastroenterol 44: 119-121, 2009.

22. Makuuchi M, Kokudo N, Arii S, et al: Development of evidencebased clinical guidelines for the diagnosis and treatment of hepatocellular carcinoma in Japan. Hepatol Res 38: 37-51, 2008.

23. Okita K: Management of hepatocellular carcinoma in Japan. J Gastroenterol 41: 100-106, 2006.

24. Ferrara N: VEGF as a therapeutic target in cancer. Oncology 69 (Suppl 3): 11-16, 2005.

25. Dowlati A, Gray R, Sandler AB, Schiller JH and Johnson DH: Cell adhesion molecules, vascular endothelial growth factor, and basic fibroblast growth factor in patients with non-small cell lung cancer treated with chemotherapy with or without bevacizumab - an Eastern Cooperative Oncology Group Study. Clin Cancer Res 14: 1407-1412, 2008.

26. Nagaoka S, Yoshida T, Akiyoshi J, et al: The ratio of serum placenta growth factor to soluble vascular endothelial growth factor receptor-1 predicts the prognosis of hepatocellular carcinoma. Oncol Rep 23: 1647-1654, 2010.

27. Yoshiji H, Kuriyama S, Yoshii J, et al: Synergistic effect of basic fibroblast growth factor and vascular endothelial growth factor in murine hepatocellular carcinoma. Hepatology 35: 834-842, 2002.

28. Wierzbowska A, Robak T, Wrzesien-Kus A, Krawczynska A, Lech-Maranda $\mathrm{E}$ and Urbanska-Rys H: Circulating VEGF and its soluble receptors sVEGFR-1 and sVEGFR-2 in patients with acute leukemia. Eur Cytokine Netw 14: 149-153, 2003.

29. Corradini SG, Morini S, Liguori F, et al: Differential vascular endothelial growth factor A protein expression between small hepatocellular carcinoma and cirrhosis correlates with serum vascular endothelial growth factor $\mathrm{A}$ and alpha-fetoprotein. Liver Int 29: 103-112, 2009.

30. Deprimo SE, Bello CL, Smeraglia J, et al: Circulating protein biomarkers of pharmacodynamic activity of sunitinib in patients with metastatic renal cell carcinoma: modulation of VEGF and VEGF-related proteins. J Transl Med 5: 32, 2007. 